Research Article

\title{
Knowledge, Attitude and Practices of Health Care Workers about Corona Virus Disease 2019 in Saudi Arabia
}

\author{
Unaib Rabbani* ${ }^{*}$, Abdullah Mohammed Al Saigul \\ Family Medicine Academy, Qassim Health Cluster, Kingdom of Saudi Arabia
}

\section{ARTICLE INFO}

\section{Article History}

Received 18 May 2020

Accepted 08 August 2020

Keywords

Attitude

COVID-19

health care worker

knowledge

practice

\begin{abstract}
Background and Objectives: Corona Virus Disease 2019 (COVID-19) pandemic is a global health emergency. Health Care Workers (HCWs) with sound knowledge and practices can help curb the pandemic. This study aimed to assess the knowledge, attitude and practices of HCWs about COVID-19 and compare physicians and non-physicians in Saudi Arabia.

Methods: An online survey was conducted among HCWs in Saudi Arabia in March and April 2020. Data were collected using a structured questionnaire having four sections including; socio-demographic and professional profile, knowledge, attitude and practices regarding COVID-19. Questionnaire link was sent through social media. Descriptive analysis was used for assessment of knowledge, attitude and practice and Chi-square test was used for comparing physicians and non-physicians. Data were analyzed using SPSS version 21.0.

Results: A total of 398 HCWs completed the questionnaire. Only $45 \%$ of the participants had correct knowledge about the agent while about $97 \%$ knew that close contact with infected person is the most important risk factor. Only $63 \%$ had correct knowledge about the role of antibiotics in COVID-19 treatment. Majority of the HCWs were worried and most frequently reported worry was risk to family. Carrying infection to home was most commonly reported fear, $92 \%$. Cleaning hands often or always was $11.5 \%$ and $87 \%$ respectively. About $71 \%$ wore mask during work. Knowledge was better among physicians compared to nonphysicians while attitude and practices were comparable between the two groups.

Conclusion: We found that there was poor knowledge about causative agent and role of antibiotics for COVID-19 but for mode of transmission and prevention there was good knowledge. There were widely prevalent worries and fears among the participants. Overall, there were good infection control practices among the HCWs. Interventions are needed to improve knowledge and address worries and fears of HCW.
\end{abstract}

(C) 2020 The Authors. Published by Atlantis Press International B.V.

This is an open access article distributed under the CC BY-NC 4.0 license (http://creativecommons.org/licenses/by-nc/4.0/).

\section{INTRODUCTION}

Increasing globalization, population and intrusion of man into new places has led to exposure of humans to unknown pathogen which results in new diseases called emerging diseases. Emerging and re-emerging diseases have always posed threat to human health. Recent emerging diseases were Sever Acute Respiratory Syndrome (SARS), Middle Ease Respiratory Syndrome (MERS), Ebola, Chikungunya, Avian flu, Swine flu and Zika. The latest among emerging diseases was discovered in Wuhan city, Hubei province of China in late December 2019 which is known as Corona Virus Diseases 2019 (COVID-19).

On December 31st 2019, World Health Organization (WHO) was informed about cases of pneumonia of unknown etiology [1]. Later the number of cases rose very rapidly and also the number of affected countries increased drastically. On January 31st 2020, WHO declared this outbreak as Public Health Emergency of international concern and later on 11th March 2020 as pandemic [2].

"Corresponding author. Email: rabbaniunaib@gmail.com

Data availability statement: Data is available from corresponding author on request.
As of April 30th 2020, more than 3.2 million cases and 234,005 deaths have been reported globally [3].

Droplet precautions are recommended by WHO for prevention of COVID-19 as there is possibility of air born transmission in aerosol generating settings [4]. Given global extent of problem and high potential of transmission, Health Care Workers (HCWs) are at higher risk of contracting COVID-19 at their work settings. A report of Chinese center for disease control and prevention (11 February 2020) indicated 1716 HCWs contracted the COVID-19 and majority, 63\% of these HCWs belonged to Wuhan where outbreak started [5].

Health care workers are the backbone of health systems. A healthy and skilled workforce is the basic requirement to ensure healthy community and this is even more important during crisis such as COVID-19 pandemic. It is also important that HCWs should have good knowledge about the disease and practice preventive measures to protect themselves and community at large. Studies from China have reported high level of knowledge among HCWs and majority of them had received training about COVID-19 [6,7]. This level of knowledge of HCWs about the disease could be one of the contributory factors behind China's exemplary control of 
the disease [8]. An understanding of status of knowledge, attitude and practices of HCWs and differences between physicians and non-physicians is an important step towards outbreak control. This would help prepare the health care workforce with targeted approaches for a better response to the pandemic.

Saudi Arabia is the Muslim pilgrimage country with millions of people across the globe visiting holy places of Makkah and Al-Madinah. Additionally there are about 10 million expatriates in the country. Saudi Arabia is no exception from the COVID-19 infection. As of April 30th 2020, a total of 24,097 cases and 169 deaths have been reported from Saudi Arabia [3]. Saudi Arabia is currently implementing a multi-sectoral national level response according to WHO operational planning guidelines [9]. To the best of our knowledge, no study has been published on assessment of knowledge of HCWs about COVID-19 in The Kingdom of Saudi Arabia. This study aimed to assess the knowledge, attitude and practices of HCWs related to COVID-19 and to compare the knowledge, attitude and practices of physicians and non-physicians in Saudi Arabia.

\section{MATERIALS AND METHODS}

\subsection{Study Setting and Population}

This was a cross-sectional survey conducted among HCWs in public and private health care facilities in Saudi Arabia from March 24th to April 15th 2020. Health care workers included; doctors, dentists, nurses, public health inspectors, technicians (laboratory, radiology and dental) and pharmacists.

\subsection{Sampling Procedure}

Convenience and snow ball sampling strategy was used to enroll potential participants. Health care workers, currently on job in Saudi Arabia were eligible to participate in the study. Questionnaire link was shared through email, WhatsApp, Facebook and Twitter by the research team. Message was also added, requesting receivers to further share the link with other co-workers in Saudi Arabia.

\subsection{Data Collection Tool and Procedure}

An online structured questionnaire with close ended questions was developed by the research team after review of literature [10-13]. Face validity of the questionnaire was done however content validity was not performed. Research team assessed all the variables for their relevance to the study objectives and contextual applicability. Questionnaire had four sections. First section was about demographic and professional characteristics. This section collected information about; age, gender, nationality, occupation and workplace. Sections 2-4 assessed knowledge, attitude and practices respectively regarding COVID-19. Section on Knowledge had 20 items related to knowledge about the disease, agent, risk factors, incubation period, symptoms, modes of transmission, role of antibiotics, antivirals and flu vaccine in the disease prevention and management, effectiveness of simple surgical mask, high risk groups for severe disease, effectives of hand washing and recommendations of masks for general population and HCWs, comparative fatality with MERS-CoV and seasonal flu, source of information and attending session on COVID-19. Section 3 about attitude had 11 questions which collected information about worriedness and fears associated with COVID-19, sufficiency of information from Ministry of Health $(\mathrm{MoH})$, difficulty in obtaining protective gears, preparation status of institute, controllability of the pandemic and accuracy of reported disease burden. Last section had five variables to collect data about practices such as washing hands, wearing surgical mask, advising and educating people about sign and symptoms of COVID-19. Questions in the knowledge section were either with specific options or "Yes/No/Don't Know", while in the attitude and practice section, 5-point likert scale either of agreement (strongly disagree, disagree, don't know, agree, strongly agree) or frequency (always, often, sometimes, occasionally, never) were used. Questionnaire was translated into Arabic language. Both Arabic and English version were provided to participants to choose language of their preference. The questionnaire is available from the link: https://forms.gle/xtP6pGqdHfto14cm6.

\subsection{Data Analysis}

Data were cleaned and assessed for completeness. Descriptive analysis was carried out to calculate frequencies and proportions for categorical and means with standard deviation for continuous variables. All the items were analyzed individually. Chi-square test was used to compare knowledge, attitude and practices of physicians and non-physicians. All HCWs including; nurses, pharmacist, technologists, technicians and health inspectors were merged as single category. $P$-value $<0.05$ was considered significant.

\subsection{Ethical Considerations}

This study was reviewed and approved by Qassim Regional Bioethics Committee (ref \#: 1441-1528621). Informed consent was obtained from all the participants before proceeding to questions in the survey. Anonymity of the participants was ensured and no personal identifier was obtained.

\section{RESULTS}

A total of 398 participants completed the questionnaire and included in the analysis. Mean age of the participants was 35.6 ( \pm 9.7$)$ years. Fifty nine percent of the participants were male. A little more than half $57 \%$ were Saudis and majority of the participants were from Qassim region (67\%). Near half (52\%) were physicians and working in Primary Health Care (PHC) centers (49.7\%) (Table 1).

Table 2 presents the knowledge, attitude and practices of HCWs about COVID-19. Only $45.5 \%$ of the participants knew about the causative agent. Almost all (97.5\%) of the respondents were aware that close contact with infected person is the most important risk factor. More than $90 \%$ of the respondents could identify common symptoms and modes of transmission. Only $63 \%$ of the respondents were aware that antibiotics have no role in treatment of COVID-19, however surprisingly, 25\% were not sure about it. 
Table 1 Socio-demographics and work characteristics of the participants $(N=398)$

\begin{tabular}{lc}
\hline Variables & $\boldsymbol{n}(\%)$ \\
\hline Gender $(n=393)$ & $232(59.0)$ \\
Male & $161(41.0)$ \\
Female & \\
Region & $266(66.8)$ \\
Qassim & $132(33.2)$ \\
Others & \\
Nationality $(n=394)$ & $224(56.9)$ \\
Saudi & $170(43.1)$ \\
Non-Saudi & \\
Occupation $(n=391)$ & $205(52.4)$ \\
Physician/Dentists & $72(18.4)$ \\
Nurse & $73(18.7)$ \\
Technologist/Health Inspector & $41(10.5)$ \\
Others & \\
Type of facility $(n=392)$ & $387(98.7)$ \\
Public sector & $5(1.3)$ \\
Private sector & \\
Place of work $(n=376)$ & $187(49.7)$ \\
PHC/Polyclinic & $121(32.2)$ \\
Hospital & $49(12.3)$ \\
Health Administration & $19(5.1)$ \\
Others & \\
Worksite $(n=375)$ & $163(43.5)$ \\
Out-patient & $62(15.6)$ \\
In-patient & $19(4.8)$ \\
Emergency Department & $131(34.9)$ \\
Others & \\
\hline
\end{tabular}

Table 2 Knowledge, attitude and practices of healthcare workers about COVID-19 $(N=398)$

\begin{tabular}{|c|c|}
\hline Variables & $n(\%)$ \\
\hline \multicolumn{2}{|c|}{ Knowledge } \\
\hline \multicolumn{2}{|c|}{ Correct knowledge about agent $(n=379)$} \\
\hline Yes & $181(45.5)$ \\
\hline No & $85(22.4)$ \\
\hline Don’t know & $113(29.8)$ \\
\hline \multicolumn{2}{|c|}{$\begin{array}{l}\text { Close contact with infected person is most important } \\
\text { risk factor }(n=393)\end{array}$} \\
\hline Yes & $383(97.5)$ \\
\hline No & $6(1.5)$ \\
\hline Not sure & $4(1.0)$ \\
\hline \multicolumn{2}{|c|}{ Incubation period of COVID-19 $(n=396)$} \\
\hline $3-5$ days & $8(2.0)$ \\
\hline 2-14 days & $366(92.0)$ \\
\hline Up to 4 weeks & $20(5.0)$ \\
\hline Not sure & $2(0.5)$ \\
\hline \multicolumn{2}{|c|}{ Most common symptoms of COVID-19 (Yes) } \\
\hline Fever $(n=392)$ & $387(98.7)$ \\
\hline Cough $(n=388)$ & $381(98.2)$ \\
\hline Shortness of breath (385) & $379(95.2)$ \\
\hline Hemoptysis $(n=280)$ & $32(11.4)$ \\
\hline Sneezing $(n=323)$ & $154(47.7)$ \\
\hline \multicolumn{2}{|c|}{ Most common modes of transmission (Yes) } \\
\hline Touching $(n=362)$ & $346(95.6)$ \\
\hline Coughing $(n=373)$ & $363(97.3)$ \\
\hline Blood transfusion $(n=254)$ & $46(18.1)$ \\
\hline Shaking hands $(n=368)$ & $368(98.7)$ \\
\hline Mosquito bite $(n=247)$ & $6(2.4)$ \\
\hline
\end{tabular}

Table 2 Knowledge, attitude and practices of healthcare workers about COVID-19 $(N=398)$-Continued

\begin{tabular}{|c|c|}
\hline Variables & $n(\%)$ \\
\hline \multicolumn{2}{|c|}{ Antibiotics are effective against COVID-19 $(n=396)$} \\
\hline Yes & $28(7.1)$ \\
\hline No & $249(62.6)$ \\
\hline Not sure & $99(25.0)$ \\
\hline Don’t know & $20(5.1)$ \\
\hline \multicolumn{2}{|c|}{ Antivirals are effective against COVID-19 $(n=395)$} \\
\hline Yes & $70(17.7)$ \\
\hline No & $141(35.7)$ \\
\hline Not sure & $146(37.0)$ \\
\hline Don’t know & $38(9.6)$ \\
\hline \multicolumn{2}{|c|}{ Flu vaccine is effective for COVID-19 $(n=395)$} \\
\hline Yes & $18(4.6)$ \\
\hline No & $272(68.9)$ \\
\hline Not sure & $81(20.5)$ \\
\hline Don’t know & $24(6.0)$ \\
\hline \multicolumn{2}{|c|}{ Past episode of flu can reduce the risk $(n=395)$} \\
\hline Yes & $24(6.1)$ \\
\hline No & $285(72.2)$ \\
\hline Not sure & $57(14.3)$ \\
\hline Don’t know & $29(7.3)$ \\
\hline \multicolumn{2}{|c|}{$\begin{array}{l}\text { In routine practice simple surgical mask is } \\
\text { effective }(n=394)\end{array}$} \\
\hline Yes & $268(68.0)$ \\
\hline No, N95 mask should be used & $102(25.6)$ \\
\hline Not sure & $24(6.1)$ \\
\hline \multicolumn{2}{|c|}{ Factors affecting severity of COVID-19 (Yes) } \\
\hline Age more than 50 years $(n=382)$ & $376(98.4)$ \\
\hline Age less than 5 years $(n=295)$ & $140(47.5)$ \\
\hline Presence of chronic diseases (382) & $380(99.5)$ \\
\hline Pregnancy $(n=332)$ & $271(81.6)$ \\
\hline Skin disorders $(n=269)$ & $32(11.9)$ \\
\hline Seasonal flu (281) & $90(32.0)$ \\
\hline \multicolumn{2}{|c|}{$\begin{array}{l}\text { Washing hands can reduce the risk if disease } \\
\text { transmission }(n=394)\end{array}$} \\
\hline Disagree & $1(0.3)$ \\
\hline Don't know & $0(0)$ \\
\hline Agree & $393(99.7)$ \\
\hline \multicolumn{2}{|c|}{$\begin{array}{l}\text { Mask is recommended to be worn by every person } \\
\text { all the time }(n=392)\end{array}$} \\
\hline Yes & $70(17.9)$ \\
\hline No & $311(79.3)$ \\
\hline Not sure & $10(2.6)$ \\
\hline Don’t know & $1(0.3)$ \\
\hline \multicolumn{2}{|c|}{$\begin{array}{l}\text { Health care workers should wear mask all the time } \\
\text { at work }(n=395)\end{array}$} \\
\hline Disagree & $91(23.0)$ \\
\hline Don't know & $12(3.0)$ \\
\hline Agree & $292(73.9)$ \\
\hline \multicolumn{2}{|c|}{ COVID-19 has high fatality than MERS-CoV $(n=394)$} \\
\hline Yes & $82(21.0)$ \\
\hline No & $309(79.0)$ \\
\hline \multicolumn{2}{|c|}{ COVID-19 has high fatality than seasonal flu $(n=391)$} \\
\hline Yes & $82(21.0)$ \\
\hline No & $309(79.0)$ \\
\hline \multicolumn{2}{|c|}{$\begin{array}{l}\text { Proportion of COVID-19 case requiring intensive care } \\
\quad(n=388)\end{array}$} \\
\hline Less than $10 \%$ & $271(68.1)$ \\
\hline Around $30 \%$ & $78(19.6)$ \\
\hline More than $50 \%$ & $39(10.1)$ \\
\hline
\end{tabular}


Table 2 Knowledge, attitude and practices of healthcare workers about COVID-19 $(N=398)$-Continued

\begin{tabular}{lc}
\hline Variables & $\boldsymbol{n}(\%)$ \\
\hline I know the concerned authority for notification $(n=387)$ & \\
$\quad$ Yes & $371(95.9)$ \\
No & $16(4.1)$ \\
Sources of information (Yes) & \\
$\quad$ Ministry of Health & $388(97.4)$ \\
Saudi CDC & $254(63.8)$ \\
WHO & $291(73.1)$ \\
US CDC & $117(29.4)$ \\
Chinese CDC & $68(17.1)$ \\
Social Media & $41(11.1)$ \\
Scientific database & $13(3.3)$ \\
Attended session on COVID-19 $(n=393)$ & \\
Yes (In-class) & $94(23.9)$ \\
Yes (Online) & $138(35.1)$ \\
No & $161(41.0)$ \\
\hline
\end{tabular}

\section{Attitude}

I am worried about COVID-19 pandemic $(n=393)$

Yes

Somewhat

$203(51.7)$

No

$188(47.8)$

$02(0.5)$

I am worried about dangers of disease $(n=323)$

Yes

$256(79.3)$

No

$67(20.7)$

I am worried about risk to my family and friends $(n=364)$

Yes

No

$360(98.9)$

I am worried about social isolation $(n=264)$

Yes

$144(54.5)$

$120(45.5)$

I am afraid of getting disease during work $(n=392)$

Disagree

Neutral

$23(5.9)$

$83(21.2)$

$286(73.0)$

I am afraid of carrying infection from my work place

to home $(n=390)$

Disagree

$12(3.1)$

$20(5.1)$

$358(91.8)$

Agree

I think available information from $\mathrm{MoH}$ is sufficient

$(n=393)$

Disagree

$11(2.8)$

$38(9.7)$

Neutral

Agree

$344(87.5)$

I find it difficult to obtain protective equipment $(n=392)$

Disagree

$196(50.0)$

Neutral

$58(14.8)$

$138(34.7)$

My institute is well prepared for COVID-19 pandemic

$(n=391)$

Disagree

Neutral

$44(11.3)$

$51(13.0)$

Agree

$296(75.7)$

In my opinion COVID-19 outbreak will $(n=391)$

Disappear completely

Continue as small epidemics in different parts

$111(28.4)$

$155(39.6)$

$125(32.0)$

(Continued)
Table 2 Knowledge, attitude and practices of healthcare workers about COVID-19 $(N=398)$-Continued

\begin{tabular}{lc}
\hline Variables & $\boldsymbol{n}(\%)$ \\
\hline I think disease burden is $(n=390)$ & \\
$\quad$ Same as being reported & $163(41.8)$ \\
Over reported & $26(6.7)$ \\
Under reported & $177(45.4)$ \\
Don't know & $24(6.2)$ \\
\hline \multicolumn{1}{c}{ Practices } & \\
\hline I clean my hands with soap or alcohol based rub $(n=391)$ & \\
Occasionally & $1(0.3)$ \\
Sometimes & $5(1.3)$ \\
Often & $45(11.5)$ \\
Always & $340(87.0)$ \\
I wear surgical mask during my work $(n=391)$ & \\
Never & $25(6.4)$ \\
Occasionally & $17(4.3)$ \\
Sometimes & $71(18.2)$ \\
Often & $117(29.9)$ \\
Always & $161(41.2)$ \\
I advise all people to seek care if they have symptoms & \\
of flu $(n=390)$ & \\
Yes & \\
No & $251(64.4)$ \\
I educate my patients about preventive measures for & $139(35.6)$ \\
COVID-19 $(n=389)$ & \\
Never & \\
Occasionally & \\
Sometimes & \\
Often & \\
Always & \\
I feel confident enough to educate my patients about & \\
COVID-19 $(n=390)$ & \\
Yes & \\
To some extent & \\
No & \\
\hline
\end{tabular}

About role of flu vaccine in prevention of COVID-19, 20\% were not sure. Regarding use of mask during routine practice, about one-third (32\%) thought that surgical mask is not suitable or were unsure about it. About use of mask, (74\%) agreed that HCWs should wear mask all the time at work. Most common sources of knowledge were $\mathrm{MoH}$ (97.4\%), WHO (73.1\%) and Saudi Center for Disease Prevention and Control (CDC) (64\%). Only 24\% and $35 \%$ of the participants had attended an in-class and online session respectively on COVID-19.

Almost all of the participants were worried about COVID-19 pandemic; most common worries were risks of contracting the infection or transmitting it to friends and family members. About one-third reported that they found it difficult to obtain personal protective equipment (PPEs).

Majority (87\%) always cleaned their hands with soap or alcohol based rub and washing hands was quite common practice. Near two-third (64\%) reported that they advised all people to seek care if there are symptoms of flu.

We also compared physicians and non-physicians with respect to their knowledge, attitude and practices (Table 3). Compared with 
Table 3 Comparison of physicians' and non-physicians' knowledge, attitude and practices regarding COVID-19 $(N=398)$

\begin{tabular}{|c|c|c|c|}
\hline Variables & Physician & $\begin{array}{c}\text { Non- } \\
\text { physician }\end{array}$ & $p$ \\
\hline \multicolumn{4}{|c|}{ Comparison of knowledge } \\
\hline \multicolumn{4}{|c|}{ Correct knowledge about agent } \\
\hline Yes & $123(62.8)$ & $56(31.5)$ & \\
\hline No & $39(19.6)$ & $45(25.3)$ & $<0.001$ \\
\hline Don’t know & $17.3(34)$ & $77(43.3)$ & \\
\hline Total & 196 & 178 & \\
\hline \multicolumn{4}{|c|}{$\begin{array}{l}\text { Close contact with infected person } \\
\text { is important risk factor }\end{array}$} \\
\hline Yes & $201(98.5)$ & $177(96.2)$ & $0.363^{*}$ \\
\hline No & $2(1.0)$ & $4(2.2)$ & \\
\hline Not sure & $1(0.5)$ & $3(1.6)$ & \\
\hline Total & 204 & 184 & \\
\hline \multicolumn{4}{|c|}{ Incubation period of COVID-19 is } \\
\hline $3-5$ days & $3(1.5)$ & $4(2.2)$ & \\
\hline $2-14$ days & $191(93.2)$ & $171(91.9)$ & $0.926^{*}$ \\
\hline Up to 4 weeks & $10(4.9)$ & $10(5.4)$ & \\
\hline Not sure & $1(0.5)$ & $1(0.5)$ & \\
\hline Total & 205 & 186 & \\
\hline \multicolumn{4}{|c|}{$\begin{array}{l}\text { Most common symptoms of } \\
\text { COVID-19 include: } \\
\text { Fever }\end{array}$} \\
\hline Yes & $203(100)$ & $179(97.3)$ & $0.024^{*}$ \\
\hline No & $0(0)$ & $2.7(5)$ & \\
\hline Total & 203 & 184 & \\
\hline \multicolumn{4}{|l|}{ Cough } \\
\hline Yes & $200(99.5)$ & $176(96.7)$ & $0.057^{*}$ \\
\hline No & $1(0.5)$ & $6(3.3)$ & \\
\hline Total & 201 & 182 & \\
\hline \multicolumn{4}{|c|}{ Shortness of breath } \\
\hline Yes & $198(98.5)$ & $176(98.3)$ & $1.000^{*}$ \\
\hline No & $3(1.5)$ & $3(1.7)$ & \\
\hline Total & 201 & 179 & \\
\hline \multicolumn{4}{|l|}{ Hemoptysis } \\
\hline Yes & $8(5.4)$ & $24(18.5)$ & 0.001 \\
\hline No & $140(94.6)$ & $106(81.5)$ & \\
\hline Total & 148 & 130 & \\
\hline \multicolumn{4}{|l|}{ Sneezing } \\
\hline Yes & $61(36.5)$ & $90(59.2)$ & $<0.001$ \\
\hline No & $106(63.5)$ & $62(40.8)$ & \\
\hline Total & 167 & 152 & \\
\hline \multicolumn{4}{|c|}{ Common modes of transmission } \\
\hline \multicolumn{4}{|l|}{ Touching } \\
\hline Yes & $179(95.2)$ & $163(96.4)$ & 0.607 \\
\hline No & $9(4.8)$ & $6(3.6)$ & \\
\hline Total & 188 & 169 & \\
\hline \multicolumn{4}{|c|}{ Coughing and sneezing } \\
\hline Yes & $192(97.5)$ & $166(97.1)$ & 1.000 \\
\hline No & $5(2.5)$ & $5(2.9)$ & \\
\hline Total & 197 & 171 & \\
\hline \multicolumn{4}{|l|}{ Blood transfusion } \\
\hline Yes & $11(8.2)$ & $34(28.8)$ & $<0.001$ \\
\hline No & $123(91.8)$ & $84(71.2)$ & \\
\hline Total & 134 & 118 & \\
\hline \multicolumn{4}{|l|}{ Shaking hands } \\
\hline Yes & $189(99.0)$ & $174(98.3)$ & $0.675^{*}$ \\
\hline No & $2(1.0)$ & $3(1.7)$ & \\
\hline Total & 191 & 177 & \\
\hline
\end{tabular}

Table 3 Comparison of physicians' and non-physicians' knowledge, attitude and practices regarding COVID-19 $(N=398)$-Continued

\begin{tabular}{lccc}
\hline Variables & Physician & $\begin{array}{c}\text { Non- } \\
\text { physician }\end{array}$ & $\boldsymbol{p}$ \\
\hline $\begin{array}{l}\text { Mosquito bite } \\
\text { Yes }\end{array}$ & $0(0)$ & $6(5.4)$ & $\mathbf{0 . 0 0 8}$ \\
No & $134(100)$ & $105(94.6)$ & \\
Total & 134 & 111 & \\
Antibiotics are effective against & & & \\
COVID-19 & & & \\
Yes & $14(6.8)$ & $13(7.0)$ & \\
No & $166(81.0)$ & $82(44.1)$ & $<\mathbf{0 . 0 0 1}$ \\
Not sure & $19(9.3)$ & $78(41.9)$ & \\
Don't know & $6(2.9)$ & $13(7.0)$ & \\
Total & 205 & 186 &
\end{tabular}

Antivirals are effective against COVID-19

$\begin{array}{lcc}\text { Yes } & 30(14.6) & 39(21.1) \\ \text { No } & 94(45.9) & 46(24.9) \\ \text { Not sure } & 66(32.2) & 77(41.6) \\ \text { Don't know } & 15(7.3) & 23(12.4) \\ \text { Total } & 205 & 185\end{array}$

$<0.001$

Flu vaccine is effective in preventing COVID-19

Yes

No

Not sure

Don’t know

Total

$$
\begin{array}{cc}
7(3.4) & 11(5.9) \\
167(81.5) & 101(54.6) \\
27(13.2) & 53(28.6) \\
4(2.0) & 20(10.8) \\
205 & 185
\end{array}
$$

$<0.001$

Having flu in the past can reduce the risk

Yes

No

Not sure

Don't know

Total

$$
\begin{array}{cc}
9(4.4) & 14(7.6) \\
168(82.0) & 113(61.1) \\
21(10.2) & 36(19.5) \\
7(3.4) & 22(11.9) \\
205 & 185
\end{array}
$$

$<0.001$

In routine practice simple surgical mask is effective

$$
\begin{array}{lcc}
\text { Yes } & 157(76.6) & 108(58.7) \\
\text { No, N95 mask should be used } & 39(19.0) & 62(33.7) \\
\text { Not sure } & 9(4.4) & 14(7.6) \\
\text { Total } & 205 & 184
\end{array}
$$

0.001

Factors affecting severity of COVID-19

Age more than 50 years

Yes

No

Total

Age $<5$ years

Yes

No

Total

Presence of chronic disease

Yes

No

Total

Pregnancy

Yes

No

Total

\begin{tabular}{ccc}
$195(98.0)$ & $176(98.9)$ & $0.688^{*}$ \\
$4(2.0)$ & $2(1.1)$ & \\
199 & 178 & \\
& & \\
$62(40.0)$ & $76(55.5)$ & $\mathbf{0 . 0 0 8}$ \\
$93(60.0)$ & $61(44.5)$ & \\
155 & 137 & \\
\multicolumn{3}{c}{} \\
$201(99.5)$ & $174(99.4)$ & 1.000 \\
$1(0.5)$ & $1(0.6)$ & \\
202 & 175 & \\
\multicolumn{3}{c}{} \\
$158(86.8)$ & $111(75.5)$ & $\mathbf{0 . 0 0 8}$ \\
$24(13.2)$ & $36(24.5)$ \\
182 & 147 & (Continued)
\end{tabular}


Table 3 Comparison of physicians' and non-physicians' knowledge,

attitude and practices regarding COVID-19 $(N=398)$-Continued

\begin{tabular}{|c|c|c|c|c|c|c|c|}
\hline Variables & Physician & $\begin{array}{c}\text { Non- } \\
\text { physician }\end{array}$ & $p$ & Variables & Physician & $\begin{array}{c}\text { Non- } \\
\text { physician }\end{array}$ & $p$ \\
\hline Skin disorders & & & & WHO & & & \\
\hline Yes & $17(11.5)$ & $15(12.6)$ & 0.780 & Yes & $184(89.8)$ & $105(56.5)$ & $<0.001$ \\
\hline No & $131(88.5)$ & $104(87.4)$ & & No & $21(10.2)$ & $81(43.5)$ & \\
\hline Total & 148 & 119 & & Total & 205 & 186 & \\
\hline Seasonal flu & & & & US CDC & & & \\
\hline Yes & $31(21.1)$ & $57(43.5)$ & $<0.001$ & Yes & $84(41.0)$ & $32(17.2)$ & $<0.001$ \\
\hline No & $116(78.9)$ & $74(56.5)$ & & No & $121(59.0)$ & $154(82.8)$ & \\
\hline Total & 147 & 131 & & Total & 205 & 186 & \\
\hline Washing hands can reduce the & & & & Chinese CDC & & & \\
\hline risk of transmission & & & & Yes & $40(19.5)$ & $28(15.1)$ & 0.245 \\
\hline Agree & $203(99.5)$ & $185(100)$ & 1.000 & No & $165(80.5)$ & $158(84.9)$ & \\
\hline Disagree & $1(0.5)$ & $0(0)$ & & Total & 205 & 186 & \\
\hline Total & 204 & 185 & & Social Media & & & \\
\hline Mask is recommended to be worn & & & & Yes & $19(9.3)$ & $24(12.9)$ & 0.251 \\
\hline by every persons all the time & & & & No & $186(90.7)$ & $162(87.1)$ & \\
\hline Yes & $24(11.8)$ & $45(24.65)$ & & Total & 205 & 186 & \\
\hline No & $173(85.2)$ & $134(72.8)$ & $0.004^{*}$ & Scientific database & & & \\
\hline Not sure & $6(3.0)$ & $4(2.2)$ & & Yes & $11(5.4)$ & $2(1.1)$ & 0.018 \\
\hline Don’t know & $0(0)$ & $1(0.5)$ & & No & $194(94.6)$ & $184(98.9)$ & \\
\hline Total & 203 & 184 & & Total & 205 & 186 & \\
\hline $\begin{array}{l}\text { Health care workers should wear } \\
\text { mask all the time at work during }\end{array}$ & & & & \multicolumn{4}{|c|}{ Comparison of attitudes } \\
\hline pandemic & & & & I am worried about COVID-19 & & & \\
\hline Disagree & $40(19.6)$ & $50(26.9)$ & 0.154 & pandemic & & & \\
\hline Don't know & $5(2.5)$ & $7(3.8)$ & & Yes & $201(99.0)$ & $184(100)$ & $0.500^{+}$ \\
\hline Agree & $159(77.9)$ & $129(69.4)$ & & No & $2(1.0)$ & $0(0)$ & \\
\hline Total & 204 & 186 & & Total & 203 & 184 & \\
\hline $\begin{array}{l}\text { COVID-19 has high fatality rate } \\
\text { than MERS-CoV }\end{array}$ & & & & $\begin{array}{l}\text { My worriedness is about dangers } \\
\text { of disease }\end{array}$ & & & \\
\hline Yes & $26(12.7)$ & $37(20.0)$ & 0.052 & Yes & $152(82.6)$ & $101(74.8)$ & 0.090 \\
\hline No & $178(87.3)$ & $148(80.0)$ & & No & $32(17.4)$ & $34(25.2)$ & \\
\hline Total & 204 & 185 & & Total & 184 & 135 & \\
\hline $\begin{array}{l}\text { COVID- } 19 \text { has high fatality rate } \\
\text { than seasonal flu }\end{array}$ & & & & $\begin{array}{l}\text { My worriedness is about risk to } \\
\text { friends and family members }\end{array}$ & & & \\
\hline Yes & $46(22.5)$ & $35(19.2)$ & 0.424 & Yes & $185(98.9)$ & $170(98.8)$ & $1.000^{*}$ \\
\hline No & $158(77.5)$ & $147(80.8)$ & & No & $2(1.1)$ & $2(1.2)$ & \\
\hline Total & 204 & 182 & & Total & 187 & 172 & \\
\hline $\begin{array}{l}\text { Proportion of COVID-19 patients } \\
\text { requiring intensive care }\end{array}$ & & & & $\begin{array}{l}\text { My worriedness is about social } \\
\text { isolation }\end{array}$ & & & \\
\hline Less than $10 \%$ & $163(80.7)$ & $103(56.9)$ & $<0.001$ & Yes & $72(51.4)$ & $69(57.5)$ & 0.327 \\
\hline Around $30 \%$ & $27(13.4)$ & $51(28.2)$ & & No & $68(48.6)$ & $51(42.5)$ & \\
\hline More than $50 \%$ & $12(5.9)$ & $27(14.9)$ & & Total & 140 & 120 & \\
\hline Total & 202 & 181 & & I am afraid of getting disease & & & \\
\hline $\begin{array}{l}\text { Know about concerned authority } \\
\text { to report suspected case }\end{array}$ & & & & during my work & & & 0.006 \\
\hline Yes & $194(97.0)$ & $172(94.5)$ & 0.224 & $\begin{array}{l}\text { Disagree } \\
\text { Neutral }\end{array}$ & $\begin{array}{c}6(3.0) \\
36(17.7)\end{array}$ & $\begin{array}{c}17(9.2) \\
44(23.9)\end{array}$ & 0.006 \\
\hline No & $6(3.0)$ & $10(5.5)$ & & Agree & $161(79.3)$ & $123(66.8)$ & \\
\hline Total & 200 & 182 & & Total & 203 & 184 & \\
\hline Sources of information & & & & I am afraid of carrying infection & & & \\
\hline Ministry of Health & & & & to my home & & & \\
\hline Yes & $200(97.6)$ & $183(98.4)$ & $0.562^{*}$ & Disagree & $2(1.0)$ & $10(5.5)$ & 0.024 \\
\hline No & $5(2.4)$ & $3(1.6)$ & & Neutral & $8(4.0)$ & $11(6.0)$ & \\
\hline Total & 205 & 186 & & Agree & $192(95.0)$ & $162(88.5)$ & \\
\hline Saudi CDC & & & & Total & 202 & 183 & \\
\hline Yes & $146(71.2)$ & $105(56.5)$ & 0.002 & & & & ontinued) \\
\hline No & $59(28.8)$ & $81(43.5)$ & & & & & \\
\hline Total & 205 & 186 & & & & & \\
\hline
\end{tabular}

Table 3 Comparison of physicians' and non-physicians' knowledge, attitude and practices regarding COVID-19 $(N=398)$-Continued

am worried about COVID-19

pandemic

No

My worriedness is about dangers of disease

Yes

No

My worriedness is about risk to friends and family members

Yes

Total

$85(98.9) \quad 170(98.8) \quad 1.000^{*}$

$2(1.1) \quad 2(1.2)$

$72(51.4) \quad 69(57.5)$

$1(42.5)$ 
Table 3 Comparison of physicians' and non-physicians' knowledge, attitude and practices regarding COVID-19 $(N=398)$-Continued

\begin{tabular}{|c|c|c|c|}
\hline Variables & Physician & $\begin{array}{c}\text { Non- } \\
\text { physician }\end{array}$ & $p$ \\
\hline \multicolumn{4}{|l|}{ I think available information from } \\
\hline Disagree & $6(3.0)$ & $5(2.7)$ & 0.312 \\
\hline Neutral & $15(7.4)$ & $22(12.0)$ & \\
\hline Agree & $182(89.7)$ & $157(85.3)$ & \\
\hline Total & 203 & 184 & \\
\hline \multicolumn{4}{|l|}{$\begin{array}{l}\text { I found it difficult to obtain } \\
\text { protective equipment }\end{array}$} \\
\hline Disagree & $118(58.1)$ & $76(41.3)$ & $<0.001$ \\
\hline Neutral & $33(16.3)$ & $24(13.0)$ & \\
\hline Agree & $52(25.6)$ & $84(45.7)$ & \\
\hline Total & 203 & 184 & \\
\hline \multicolumn{4}{|l|}{$\begin{array}{l}\text { My institute is well prepared for } \\
\text { COVID-19 pandemic }\end{array}$} \\
\hline Disagree & $19(9.4)$ & $25(13.7)$ & 0.072 \\
\hline Neutral & $33(16.3)$ & $17(9.3)$ & \\
\hline Agree & $151(74.4)$ & $141(77.0)$ & \\
\hline Total & 203 & 183 & \\
\hline \multicolumn{4}{|l|}{$\begin{array}{l}\text { In my opinion COVID-19 } \\
\text { outbreak will }\end{array}$} \\
\hline Disappear completely & $43(21.4)$ & $65(35.3)$ & 0.008 \\
\hline Continue as small epidemics & $85(42.3)$ & $68(37.0)$ & \\
\hline Shrink to sporadic cases & $73(36.6)$ & $51(27.7)$ & \\
\hline Total & 201 & 184 & \\
\hline \multicolumn{4}{|l|}{ I think disease burden is } \\
\hline Same as being reported & $79(39.1)$ & $80(44.0)$ & 0.075 \\
\hline Over reported & $11(5.4)$ & $14(7.7)$ & \\
\hline Under reported & $104(51.5)$ & $73(40.1)$ & \\
\hline Don’t know & $8(4.0)$ & $15(8.2)$ & \\
\hline Total & 202 & 182 & \\
\hline
\end{tabular}

Comparison of practices

\begin{tabular}{|c|c|c|c|}
\hline $\begin{array}{l}\text { I clean my hands } \\
\text { alcohol rub }\end{array}$ & & & \\
\hline Occasionally & $0(0.0)$ & $1(0.5)$ & \\
\hline Sometimes & $3(1.5)$ & $2(1.1)$ & 0.056 \\
\hline Often & $31(15.3)$ & $14(7.7)$ & \\
\hline Always & $169(83.3)$ & $166(90.7)$ & \\
\hline Total & 203 & 183 & \\
\hline $\begin{array}{l}\text { I wear surgical ca } \\
\text { my work }\end{array}$ & & & \\
\hline Never & $8(3.9)$ & $17(9.3)$ & \\
\hline Occasionally & $8(3.9)$ & $9(4.9)$ & 0.075 \\
\hline Sometimes & $32(15.8)$ & $39(21.3)$ & \\
\hline Often & $63(31.0)$ & $53(29.0)$ & \\
\hline Always & $92(45.3)$ & $65(35.5)$ & \\
\hline Total & 203 & 183 & \\
\hline $\begin{array}{l}\text { I advise all peopl } \\
\text { they have sym }\end{array}$ & & & \\
\hline Yes & $121(59.9)$ & $127(69.4)$ & 0.052 \\
\hline No & $81(40.1)$ & $56(30.6)$ & \\
\hline Total & 202 & 183 & \\
\hline $\begin{array}{l}\text { I educate my pat } \\
\text { preventive med } \\
\text { COVID-19 }\end{array}$ & & & \\
\hline Never & $0(0)$ & $5(2.7)$ & \\
\hline Often & $0(0)$ & $3(1.6)$ & 0.021 \\
\hline
\end{tabular}

Table 3 Comparison of physicians' and non-physicians' knowledge, attitude and practices regarding COVID-19 $(N=398)$-Continued

\begin{tabular}{lccc}
\hline Variables & Physician & $\begin{array}{c}\text { Non- } \\
\text { physician }\end{array}$ & $\boldsymbol{p}$ \\
\hline Sometimes & $11(5.4)$ & $13(7.1)$ & \\
Occasionally & $49(24.3)$ & $33(18.1)$ & \\
Always & $142(70.3)$ & $128(70.3)$ & \\
$\quad$ Total & 202 & 182 & \\
I feel confident enough to educate & & & \\
my patients about COVID-19 & & & \\
Yes & $157(77.7)$ & $138(75.4)$ & $0.679^{*}$ \\
To some extent & $42(20.8)$ & $40(21.9)$ & \\
No & $3(1.3)$ & $5(2.7)$ & \\
Total & 202 & 183 & \\
\hline
\end{tabular}

"Fisher exact $p$-value.

non-physicians, physicians generally showed better knowledge in most of the studied items. The differences in identifying the causative agent, role of antibiotics, flu vaccine, fear of getting disease at work place and carrying infection to home were statistically significant, while there was no significant differences for other items such as; identifying main modes of transmission, factors that increases the severity of the disease, worriedness about COVID-19 and hand washing and wearing mask practices. About effectiveness of antibiotics, correct answer was given by $81 \%$ physicians and $44 \%$ non-physicians $(p$-value $<0.001)$. There was no significant difference between the two groups with respect to worriedness about COVID-19. However, fear of getting disease at work place and carrying infection to home were higher among physicians, 79\% and $95 \%$ respectively compared to non-physicians, $67 \%$ and $88 \%$ respectively. With respect to practices, we did not find significant difference between physicians and non-physicians. Around $8 \%$ of the physicians and 35\% of non-physicians never or occasionally used mask during their work. These respondents were mainly working in administrative and non-patient care areas.

\section{DISCUSSION}

This study, to the best of our knowledge is the first of its kind from Saudi Arabia to assess knowledge, attitude and practices of HCWs in the Kingdom.

Correct knowledge about a disease is an important factor in prevention and control of disease [14] this is even more important during pandemics where a large section of population is susceptible. In our study, knowledge about agent was low $45 \%$. This is lower than studies from China [7] and Pakistan [15] where 99\% and 100\% respondents were correct. However, in these studies researchers inquired only if this was a viral disease, whereas in our study we inquired about the name of the specific virus. Knowledge about close contact as most important risk factor was higher in our study (97\%) compared to HCWs in China (67\%) [7]. Knowledge about incubation period was slightly lower 92\% than reported from Pakistan $96 \%$ [15]. Our study found that correct knowledge about role of antibiotics was $63 \%$ which is higher than reported from China $58 \%$ [7] but lower than Pakistan 82\% [15]. Having more than one third of participants with incorrect knowledge about the antibiotics use for COVID-19 indicates a poor state of basic understanding of 
infectious diseases. This has important implications on patients as well as health care system in terms of health consequences such as antimicrobial resistance and costs of care.

Previous studies from Saudi Arabia and Greece also reported a higher proportion of HCWs being worried about their families during MERS-CoV and H1N1 epidemics respectively [11,13] Risk to friends and family members was most frequent reason in our study, a finding similar to earlier report from China during this pandemic $[7,16]$. Majority $(75 \%)$ of the HCWs believed that their institution was not prepared for COVID-19 pandemic. Similar findings were reported previously from Saudi Arabia during MERS-CoV outbreak [10]. This is also reflected as fear among 92\% of the respondents about carrying infection to their homes. This is important finding as one's beliefs about preparedness of his/her institution has effects on feelings of safety, motivation and morale during work [17]. Proper risk communication with HCWs and their training is essential in this regard along with upgrading and maintaining health care facilities to face the challenge of emerging diseases such as COVID-19.

Without adequate practices, knowledge and attitude do not provide the desired outcome for prevention and control of diseases. In our study we found that a large proportion frequently cleaned their hands. This finding is similar to a study from Pakistan [15] while a lower proportion $82 \%$ of HCWs reported cleaning their hands often or always following MERS-CoV outbreak in Saudi Arabia [10]. This may indicate improvement in hygiene practice which has resulted from previous experience of an outbreak. For a respiratory disease, wearing mask during practice is an effective measure for prevention of infection among HCWs. Our study found that about $30 \%$ often and $41 \%$ always used surgical mask at work. This finding is similar to previous study from Saudi Arabia where 24\% and 43\% of HCWs used mask often and always respectively [10]. We assume that even small proportion of workers who do not practice can pose risk to other colleagues and patients.

We also compared knowledge, attitudes and practices between physicians and non-physicians. The findings are similar to previous study from Saudi Arabia where physicians were found to have better knowledge about MERS-CoV than other HCWs [10]. Generally, it was observed that knowledge about agent, roles of antibiotics and antivirals and use of masks were better among physicians compared to non-physicians. However there was no significant difference in knowledge between the two groups with respect to common signs and symptoms, modes of transmission, factors affecting severity of the disease and concerned authority for reporting a case.

There was no significant difference between physicians and non-physicians in terms of worriedness. However, fear of getting disease during work and carrying infection to home was higher among physicians. There were no significant differences in the practices of both types of HCWs except for educating patients about prevention and control of COVID-19, which was higher among physicians. These differences could be due to the fact that in our sample non-physicians also included technicians, health inspectors and those working in administration and they are not in direct contact with the patient. This may have led to perception of lower risk of getting infected and different opportunities for educating patients. We also did exploratory analysis (results not shown) by excluding technicians and health inspectors and compared physician with nurses. This exercise however did not show any major changes in the results.

Poor knowledge about causative agent, role of antibiotics and antiviral medications and wide spread fears need to be addressed. Policy makers and administrators should arrange workshops and training sessions for the staff. Health care workers need to avoid information from unauthentic sources and refer to only official communications from their ministry and/or other recognized international organizations such as CDC and WHO. Social media is known source of misinformation and may lead to myths and malpractices [18]. Therefore HCWs should not give any heed to such information. Psychological assessment and counselling are also required to protect the mental health of frontline workers.

In this study we comprehensively assessed the knowledge, attitude and practices of a diverse group of HCWs. However there are certain limitations which should be considered while interpreting findings of this study. First, given the COVID-19 pandemic and lockdown across the country, face-to-face interviews were not possible, therefore we designed this study as online survey in which it is possible that some of the respondents might have not understood the question properly and may not answer accurately. This however, we assume to be affecting our results minimally because we developed questionnaire based on previous studies specifically from Saudi Arabia and we also translated questionnaire to Arabic language for better understanding by some respondents. Second, in our sample there was over representation of participants from Qassim region. This, we assume be a minor limitation as there is unified response at the country level for the prevention and control of COVID-19. There are standardized protocols and guidelines from $\mathrm{MoH}$ and Saudi CDC which are implemented across the country uniformly. Third, this was an open online survey where response rate cannot be ascertained. It is also possible that those responded might be different from those who did not respond despite receiving the survey link. This may limit the generalizability of our study. We did not do a priori sample size calculation given the open nature of survey and defined time period of data collection (3 weeks). However, we are able to reach a sample size which would give us absolute precision ranging from $1.5 \%$ to $5.0 \%$. Additionally, composition of our sample with respect to gender and nationality among physicians and non-physicians was comparable to national health workforce statistics [19]. Finally, as the epidemic continues, more disease facts are evolving and staff knowledge and practices are changing.

\section{CONCLUSION}

We found the knowledge about the agent of the disease and role of antibiotics was poor among HCWs in Saudi Arabia which needs attention of policy makers. However, knowledge about the other aspects of disease such as modes of transmission and prevention was better. Worriedness and fears were present among majority of the HCWs which may affect their level of motivation. Infection control practices such as cleaning hands, wearing mask and educating people about the disease were also high. These findings call for targeted interventions such as timely orientation about emerging diseases, training on disease management and counseling services for worries and fears. Ensuring adequate infection control supplies and constructive supervision of staff practices can augment the overall performance. 


\section{CONFLICTS OF INTEREST}

The authors declare they have no conflicts of interest.

\section{AUTHORS' CONTRIBUTION}

UR conceived the research idea and developed the proposal. Both UR and AMAS developed the study tool. UR analyzed the data and AMAS reviewed the results with UR. UR wrote the first draft and both edited the draft for final submission. Both the authors approved final version for publication.

\section{FUNDING}

No financial support was provided.

\section{REFERENCES}

[1] World Health Organization. Novel coronavirus - China. 2020. Available from: https://www.who.int/csr/don/12-january-2020-novel-coronavirus-china/en/ [cited March 16, 2020].

[2] World Health Organization. Novel coronavirus (2019-nCoV) situation report-51. Geneva, Switzerland: World Health Organization; 2020.

[3] Saudi Center for Disease Prevention and Control. COVID-19: daily updates Riyadh, KSA. 2020. Available from: https://covid19. cdc.gov.sa/daily-updates/ [cited April 30, 2020].

[4] World Health Organization. Modes of transmission of virus causing COVID-19: implications for IPC precaution recommendations. Scientific brief. Geneva, Switzerland: World Health Organization; 2020. Available from: https://www.docdroid.net/ Xc5kEn7/who-2019-ncov-sci-brief-transmission-modes-20202eng.pdf [cited April 29, 2020].

[5] Vital surveillances. The epidemiological characteristics of an outbreak of 2019 novel coronavirus diseases (COVID-19) - China, 2020. China CDC Weekly 2020;2;113-22.

[6] Shi Y, Wang J, Yang Y, Wang Z, Wang G, Hashimoto K, et al. Knowledge and attitudes of medical staff in Chinese psychiatric hospitals regarding COVID-19. Brain Behav Immun Health 2020;4;100064.

[7] Huynh G, Nguyen TNH, Tran VK, Vo KN, Vo VT, Pham LA. Knowledge and attitude toward COVID-19 among healthcare workers at District 2 Hospital, Ho Chi Minh City. Asian Pac J Trop Med 2020;13;260-5.
[8] World Health Organization. Report of the WHO-China joint mission on coronavirus disease 2019 (COVID-19). 2020. Available from: https://www.who.int/docs/default-source/coronaviruse/who-china-joint-mission-on-covid-19-final-report.pdf [cited April 29, 2020].

[9] World Health Organization. COVID-19 strategic preparedness and response plan: operational planning guidelines to support country preparedness and response. Geneva, Switzerland: World Health Organization; 2020.

[10] Alsahafi AJ, Cheng AC. Knowledge, attitudes and behaviours of healthcare workers in the Kingdom of Saudi Arabia to MERS coronavirus and other emerging infectious diseases. Int J Environ Res Public Health 2016;13;1214.

[11] Asaad A, El-Sokkary R, Alzamanan M, El-Shafei M. Knowledge and attitudes towards Middle East respiratory syndrome-coronavirus (MERS-CoV) among health care workers in south-western Saudi Arabia. East Mediterr Health J 2020;26;435-42.

[12] Puri S, Singh A, Koushal V, Thakare M, Singhal A. Knowledge, attitude and practice regarding the H1N1 pandemic amongst healthcare providers, and preparedness in a multispeciality teaching hospital in North India. Public Health 2011;125;795-8.

[13] Goulia P, Mantas C, Dimitroula D, Mantis D, Hyphantis T. General hospital staff worries, perceived sufficiency of information and associated psychological distress during the $\mathrm{A} / \mathrm{H} 1 \mathrm{~N} 1$ influenza pandemic. BMC Infect Dis 2010;10;322.

[14] McEachan R, Taylor N, Harrison R, Lawton R, Gardner P, Conner M. Meta-analysis of the reasoned action approach (RAA) to understanding health behaviors. Ann Behav Med 2016;50;592-612.

[15] Saqlain M, Munir MM, Rehman SU, Gulzar A, Naz S, Ahmed Z, et al. Knowledge, attitude, practice and perceived barriers among healthcare workers regarding COVID-19: a cross-sectional survey from Pakistan. J Hosp Infect 2020;105;419-23.

[16] Zhang M, Zhou M, Tang F, Wang Y, Nie H, Zhang L, et al. Knowledge, attitude and practice regarding COVID-19 among healthcare workers in Henan, China. J Hosp Infect 2020;105;183-7.

[17] Cai H, Tu B, Ma J, Chen L, Fu L, Jiang Y, et al. Psychological impact and coping strategies of frontline medical staff in Hunan between January and March 2020 during the outbreak of coronavirus disease 2019 (COVID-19) in Hubei, China. Med Sci Monit 2020;26:e924171.

[18] Depoux A, Martin S, Karafillakis E, Preet R, Wilder-Smith A, Larson $\mathrm{H}$. The pandemic of social media panic travels faster than the COVID-19 outbreak. J Travel Med 2020;27;taaa031.

[19] Ministry of Health. Annual statistical book, Saudi Arabia. Riyadh, Saudi Arabia: Ministry of Health; 2017. Available from: https:// www.moh.gov.sa/en/Ministry/Statistics/book/Documents/ Statistical-Yearbook-1437H.pdf. 